\title{
Retinal vascular changes in congenital hypertrophy of the retinal pigment epithelium
}

\author{
P. E. CLEARY, Z. GREGOR, AND A. C. BIRD \\ From Moorfields Eye Hospital and the Institute of Ophthalmology, London
}

Congenital hypertrophy of the retinal pigment epithelium (CHRPE) is a well-defined clinical entity which is usually an incidental finding during routine fundus examination (Buettner, 1975). Recent reports have emphasized the characteristic features that distinguish it from malignant melanoma of the choroid and other pigmented fundus lesions (Buettner, 1975; Purcell and Shields, 1975). Stereoscopic fundus photography and fluorescein angiography are useful in confirming the diagnosis (Buettner, 1975). The histopathology is that of hypertrophy of a single layer of retinal pigment epithelial cells with an increase in the concentration of pigment granules within the cells, but whether this pigment is lipofuscin or melanin it has not been established (Kurz and Zimmerman, 1962; Buettner, 1975). The photoreceptor cells overlying the hypertrophic pigment epithelium degenerate and disappear, thus providing a morphological basis for accompanying visual field defects (Kurz and Zimmerman, I962; Buettner, 1975). Intraretinal pigment migration is present in a minority of cases, but other abnormalities of the inner retina have not been previously identified. This report presents five cases with abnormalities in the retinal vasculature overlying areas of CHRPE.

\section{Patients}

The patients' ages ranged from 42 to 64 years. None had evidence of previous or other ocular disease. All were examined for signs of systemic disease but no abnormalities were found. Routine investigations were within normal limits and included a full blood count, chest $x$-ray examination, and electrocardiogram.

\section{Clinical findings}

\section{OPHTHALMOSCOPY}

The fundus lesions were solitary, round, flat, hyperpigmented, and well demarcated. They varied in colour from greyish-brown to black and in size from $I$ to about 8 disc diameters. Characteristic lacunae were Road, London ECIV 2PD present in all, and the typical hypopigmented haloes surrounded four lesions. Abnormalities of the overlying retinal vessels were seen in only two cases, with gross attenuation of a retinal artery in one and sheathing of a vein in another. A discrete, hyperpigmented and slightly raised area about $I$ disc diameter in size was observed in the centre of the lesion in one case (Fig. I).

\section{FLUORESCEIN ANGIOGRAPHY}

Overlying retinal vascular abnormalities were demonstrated in all the lesions. In one there was no perfusion of the overlying retinal vessels (Fig. 2) and in another perfusion was absent except for delayed filling of a single retinal vein with late staining of its wall (Fig. 3). Gross attenuation of a retinal artery and its branches occurred in the centre of the lesion in one patient (Fig. 4). The remaining two patients gave a complicated appearance of retinal capillary closure with leakage of fluorescein from abnormal capillaries. In one leaking retinal capillaries and venules in the centre of the lesion were related to areas of retinal capillary closure or were present at the edge of non-perfused retina (Fig. 5). In the other an abnormal leaking capillary network corresponded to a hyperpigmented raised area in the centre of the lesion (Fig. I). This capillary network filled in the earliest stages of the fluorescein angiogram when the surrounding retinal vessels were not perfused, thus implying an arterial supply from the choroid.

\section{VISUAL FIELDS}

Four patients were examined by Tubingen perimetry. In all four absolute scotomas corresponded to the site of the fundus lesions.

\section{Discussion}

The fundus lesions in these five patients correspond with previous descriptions of congenital hypertrophy of the retinal pigment epithelium (Reese and Jones, 1956; Reese, I960; Kurz and Zimmerman, 1962; Hogan and Zimmerman, 1962 ; Buettner, 1975; Purcell and Shields, 1975). However, abnormalities of the overyling retinal vasculature have not before been identified. Retinal blood vessels have been described particularly as undeviated and normal (Buettner, 1975). The vascular 

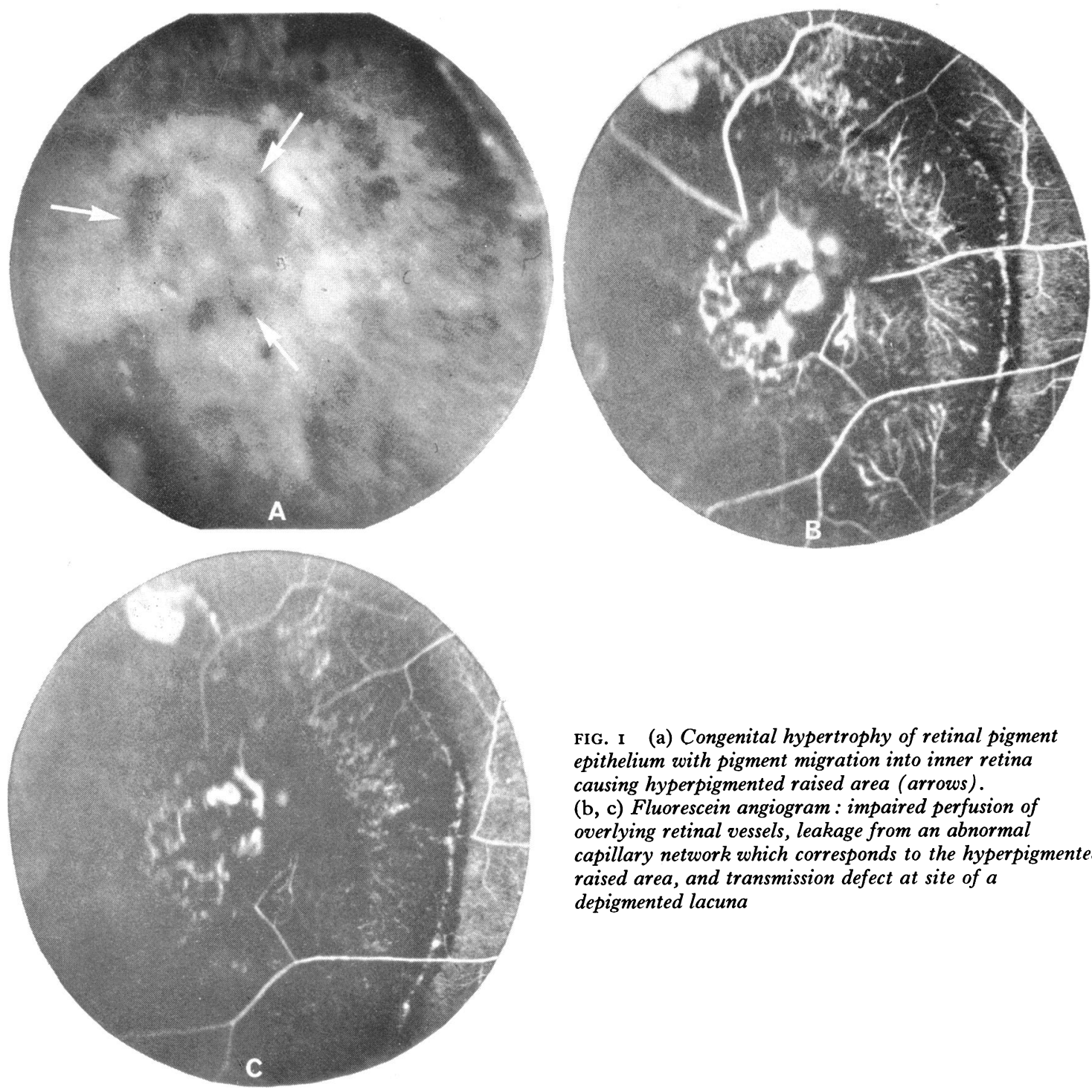

FIG. I (a) Congenital hypertrophy of retinal pigment epithelium with pigment migration into inner retina causing hyperpigmented raised area (arrows).

(b, c) Fluorescein angiogram : impaired perfusion of overlying retinal vessels, leakage from an abnormal capillary network which corresponds to the hyperpigmented raised area, and transmission defect at site of a depigmented lacuna

changes in all our cases were defined by fluorescein angiography and consisted of vascular non-perfusion with obliteration of the retinal capillary bed. In addition, two cases had retinal capillary leakage, which in one was from presumed intraretinal new vessels. The configuration of the leaking capillary network in this second case (Fig. I) suggested that the blood vessels were derived from the choroid. The associated pigment migration into the inner retina may well have been accompanied by fibrovascular ingrowth from subretinal tissues.

Photoreceptor degeneration in CHRPE is probably progressive, since the consequent visual field loss becomes denser with increasing age (Kurz and

Zimmerman, 1962; Buettner, 1975). By contrast, the inner retina remains well preserved. The absolute scotomas demonstrated by Tubingen perimetry indicate that there was severe receptor cell loss in these patients with obliteration of overlying retinal capillaries.

Precisely similar retinal vascular abnormalities have been described in retinitis pigmentosa (Duke Elder, 1967; Noell, 1953), in the retinae of rats with hereditary visual cell degeneration (Gerstein and Dantzker, 1969), in light-damaged rat retinae (Dantzker and Gerstein, 1969), and after argon and ruby laser photocoagulation of monkey retinae (Okisaka, Kuwabara, and Aiello, 1975), in which 


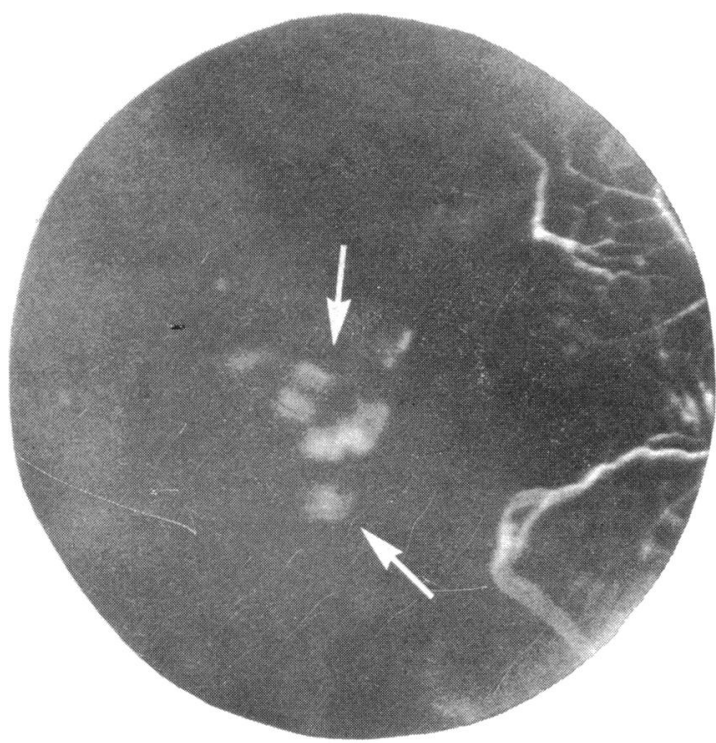

Fig 2

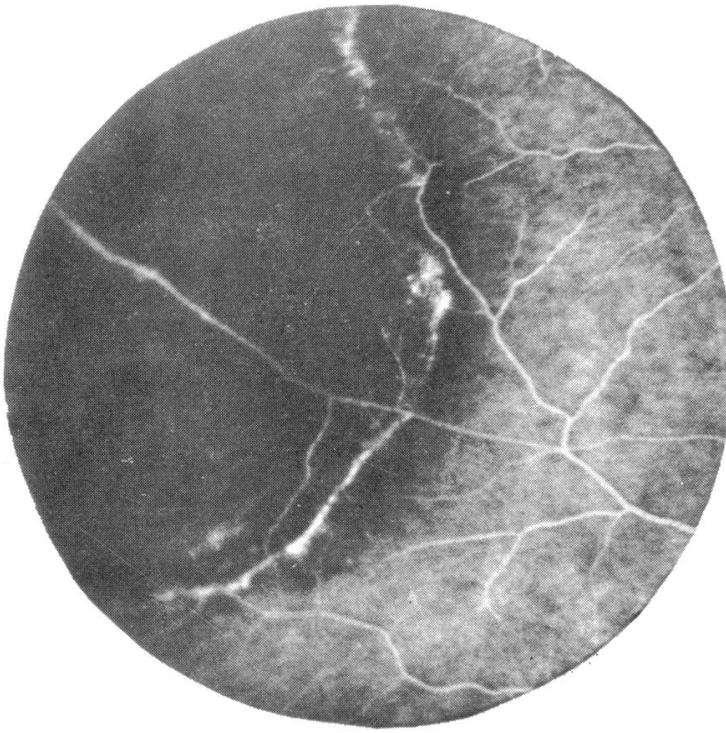

Fig 3

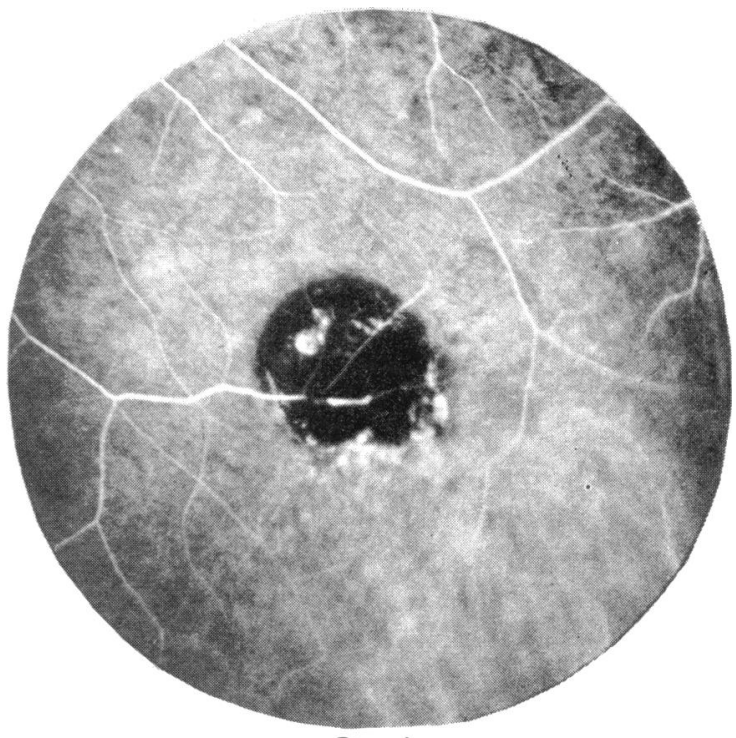

Fig 4

FIG. 2 Fluorescein angiogram of congenital hypertrophy of retinal pigment epithelium showing no perfusion of overlying retinal vessels but with transmission defects at site of depigmented lacunae (arrows)

FIG. 3 Fluorescein angiogram of congenital hypertrophy of retinal pigment epithelium : no perfusion of overlying retinal vessels except for filling of a single vein

FIG. 4 Fluorescein angiogram of congenital hypertrophy of retinal pigment epithelium with attenuation of a retinal artery and its branches, typical lacunae, and hyperfluorescent lower border

conditions there is combined degeneration of photoreceptors and retinal pigment epithelium. Obliteration of retinal capillaries was also observed in cats' eyes when iodoacetate was used to destroy selectively the photoreceptor layer alone, indicating that the presence of an intact retinal pigment epithelium did not seem to modify the vascular response to outer retinal atrophy (Dantzker and Gerstein, 1969).

In some of these conditions obliteration of retinal capillaries might possibly result from secondary changes in the inner retina after outer retinal

atrophy, and in light-damaged rat retinae the most severe vascular changes coincided with considerable distortion of the inner retinal layers (Dantzker and Gerstein, 1969). However, these authors observed that the early stages of vascular degeneration preceded inner layer disease and they noted a close temporal relationship between the progressive degeneration with thinning of the outer retinal layers and the disappearance of retinal capillaries. They concluded that retinal capillary degeneration occurred as a result of outer retinal loss and was not 


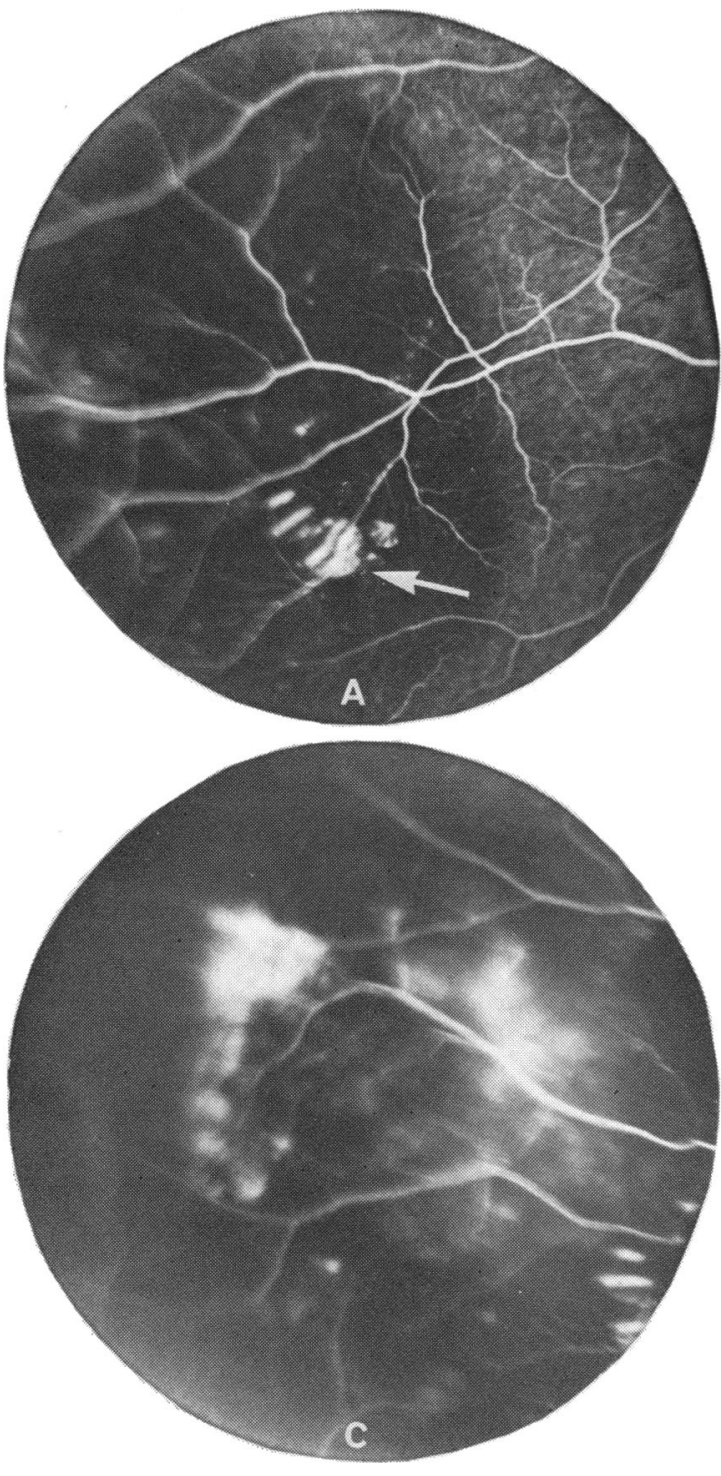

directly related to the state of the inner retina (Gerstein and Dantzker, 1969).

It has been argued that outer retinal atrophy would result in an increased oxygen concentration in the inner retina because of the combined effect of reduced metabolic requirements by the outer retina and closer apposition of the inner retina to the choroid (Noell, 1953; Gerstein and Dantzker, 1969). Such an increase in oxygen tension caused vasoconstriction of retinal arterioles and veins (Dollery, Hill, Mailer, and Sousa Ramalho, 1964), and this may account for obliteration of retinal vessels in the presence of outer retinal atrophy (Gerstein and Dantzker, 1969).

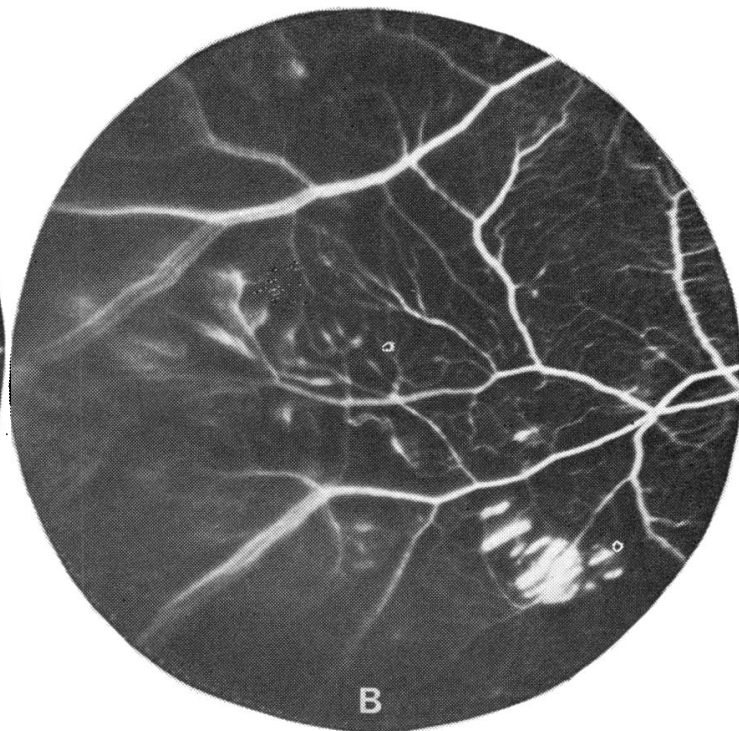

FIG. 5 Fluorescein angiogram of congenital hypertrophy of retinal pigment epithelium. (a) Masking of background choroidal fluorescence, except at site of a lacuna (arrow). (b) Abnormal retinal capillaries and areas of retinal capillary closure. (c) Later stages : leakage from abnormal vessels at edge of non-perfused retina

This does not completely explain the retinal vascular occlusion associated with outer retinal atrophy. Mature retinal vessels are known to be remarkably immune to high concentrations of oxygen and, moreover, retinal capillary obliteration has not been successfully produced in adult animals exposed to high concentrations of oxygen, although in these animals a high oxygen tension was maintained for only a few weeks (Ashton, 1966, 1976). However, obliteration of retinal arterioles due to gross thickening of their walls has been observed histopathologically in cases of retinal dystrophy (Ashton and Tripathi, 1976), and possibly a prolonged high oxygen concentration might cause 
arteriolar constriction with a reduced blood flow to more distal tissues producing significant ischaemia. This could certainly account for the retinal capillary closure and leakage overyling CHRPE, which are reminiscent of the changes in branch or central retinal vein occlusion-changes usually assumed to imply retinal ischaemia rather than a high oxygen tension.

\section{Summary}

The overlying retinal blood vessels were abnormal in five cases of congenital hypertrophy of the retinal pigment epithelium. This illustrated the wellrecognized association between outer retinal degeneration and obliteration of the overlying retinal vasculature. The proposed pathophysiological mechanisms, however, seem inadequate to explain completely the morphological changes of the retinal blood vessels in the presence of atrophy of the outer retina.

We thank Mr K. S. Sehmi for his photographic expertise and Miss J. Fisher for secretarial assistance.

\section{References}

AShton, N. (1966) Amer. F. Ophthal., 62, 412

(1976) Personal communication

and TRIPATHI, R. (1976) Personal communication

BUETTNER, H. (1975) Amer. F. Ophthal., 79, 177

DANTZKER, D. R., and GERSTEIN, D. D. (1969) Arch. Ophthal., 81, i०6

DOLlERY, C. T., HILl, D. W., MAILER, C. M., and SOUSA RAMALHO, P. (1964) Lancet, 2, 29 I

DUKE ELDER, s. (1967) 'System of Ophthalmology', vol. ıo, pp. 577-606. Kimpton, London

GERSTEIN, D. D., and DANTZKER, D. R. (1969) Arch. Ophthal., 81, 99

hogan, M., and zimmerman, L. E., eds (1962) 'Ophthalmic Pathology: an atlas and textbook', 2 nd ed. p. 477.

Saunders, Philadelphia

KURZ, G. H., and ZIMMERMAN, L. E. (1962) Int. ophthal. Clin., 2, 44I

NOELL, W. K. (1953) Studies on the electrophysiology and metabolism of the retina, USAF Report No. 2 I/1 $201 / 0004$ okisaka, s., kUWABara, T., and aiello, L. M. (1975) Amer. F. Ophthal., 80, 591

PURCELl, J. J. JR., and SHIEldS, J. A. (1975) Arch. Ophthal., 93, I 122

REESE, A. B. (1960) Amer. Э. Ophthal., 50, ro66 , and JONES, I. S. (1956) Ibid., 42, 207 